\title{
The diagnosis and management of patients admitted to hospital with acute breathlessness
}

\author{
S. B. PEARSON \\ D.Phil., M.R.C.P.
}

\author{
Elizabeth M. Pearson \\ M.B. B.S., D.C.H., M.R.C.G.P.
}

\author{
J. R. A. Mitchell \\ D.Phil., M.D., F.R.C.P. \\ Department of Medicine, University Hospital, Nottingham NG7 2UH
}

\begin{abstract}
Summary
The authors have studied 352 emergency medical admissions during a summer period and 355 during the winter months to identify 177 patients who had been treated for breathlessness. Multiple and rapidly changing treatments have been taken to indicate diagnostic uncertainty. Using this model, the authors have shown that there is much more uncertainty in winter than in summer and that the working diagnosis of chest infection or asthma is particularly likely to be associated with multiple treatment. It is questionable whether the stereotyped descriptions of diseases in standard text books provide an adequate basis for emergency treatment decisions and it is considered that the diagnostic value of investigations such as chest radiography needs further careful scrutiny.
\end{abstract}

\section{Introduction}

Breathlessness is a common medical emergency and can arise from a variety of conditions which require totally different initial treatment. The authors found it very difficult confidently to differentiate between heart failure, chest infection, asthma and pulmonary embolism and, as the majority of acute medical admissions are initially diagnosed and treated by recently qualified doctors, they wondered whether the training of the latter was providing them with better diagnostic criteria.

Textbooks which are widely used by undergraduates (Macleod, 1977; Houston, Joiner and Trounce, 1975) describe very clearly how to treat bronchopneumonia, left ventricular failure, asthma and embolization without making it equally clear how one can differentiate between them. One reads that crepitations can occur in heart failure or infection, that neck vein engorgement can be found in both heart failure and pulmonary embolization and that patients with any of the common causes of breathlessness may wheeze. Fever is an accompaniment of myocardial infarction and pulmonary embolism as well as infection, while sputum purulence may be due to the presence of the eosinophils of asthma as well as to the pus cells of infection (Crofton and Douglas, 1975).

At the bedside, it is thus difficult to differentiate between the major causes of dyspnoea; to ensure that relief is given as quickly as possible, a doctor therefore may initiate treatment for more than one disease. While multiple pathology undoubtedly exists in some patients, it was the authors' belief that in the majority, multiple treatment reflects an inability to reach a precise diagnosis.

Diagnostic labels and treatment, once initiated, tend to be accepted. Whilst it is common for additional treatment to be given during the hospital admission, initial decisions to use antibiotics, bronchodilators, diuretics or anticoagulants are seldom reversed. If the initial label is incorrect, then inappropriate treatment may be continued. The authors therefore studied the way in which doctors who initiate treatment for breathessness make their decisions and have attempted to identify the signs and symptoms on which they rely most.

\section{Methods}

Diagnosis and treatment on admission

All patients admitted as medical emergencies to the General Hospital, Nottingham, were studied during 2 periods, one in summer and one in winter. The first was from 22 August 1976 to 20 September 1976, and the second was from 29 November to 24 December 1976. During the first period, 352 patients were admitted and during the second, 355.

Each day, all new medical admissions were seen by S.B.P. and breathless patients who had been treated for heart failure, chest infection, asthma and pulmonary embolism were identified for inclusion in the survey. These groups were based upon the initial treatment given and were therefore the 
admitting clinician's 'operational diagnoses'. The following definitions were used:

Heart failure. The initiation of treatment with digoxin, diuretics or the prescribing of an increased dose of diuretic was taken to indicate an operational diagnosis of heart failure, except for patients given digoxin as an anti-dysrhythmic drug, and patients given low-dose diuretics as part of an anti-hypertensive regime, who were excluded.

Chest infection. The prescription of an antibiotic was taken to indicate an operational diagnosis of chest infection.

Asthma. The prescription of bronchodilators or steroids (oral, parenteral or inhaled) was taken to indicate an operational diagnosis of asthma.

Pulmonary embolus. The initiation of anticoagulation in a breathless patient was taken to indicate an operational diagnosis of pulmonary embolism.

Aminophylline is of value in both heart failure and asthma so its use may reflect uncertainty on the part of the prescribing doctor. If it was prescribed alone or in combination with steroids this was taken to indicate a working diagnosis of asthma; prescription in combination with a diuretic was taken to indicate heart failure and in combination with an antibiotic, asthma plus chest infection.

\section{Subsequent diagnosis and treatment}

All acute medical admissions, whether initially included in the survey or not, had their treatment charts inspected every day by S.B.P. so that change in diagnosis and treatment category during ade mission could be recorded and so that patients whe were first treated for breathlessness during theip? subsequent stay could be added to the survey.

\section{Evidence used by admitting clinicians to make operational diagnoses}

The case notes of all patients included in the surve were inspected for the presence of the physical signs. and symptoms set out in Table 1 . The absence of a symptom or physical sign from the case notes was taken to mean that it had not been elicited by the admitting doctor.

Where chest X-rays were done on admission the doctor who initiated treatment was asked whethej the X-ray was done before treatment was initiate and, if so, whether it helped him to make his treateo ment decision.

\section{Statistical methods}

The treatment groups were compared in respect of the symptoms and signs recorded in Table 1 . Using a $2 \times 2$ contingency table and applying the $\chi^{2}$ test each symptom and sign was tested to see whethes ito was more common in one treatment group than another. Where numbers were small, Yates' core rection (Yates, 1934) was applied as recommen

TABLE 1. Pro forma used to extract information from medical notes

\begin{tabular}{ll}
\multicolumn{1}{c}{ Symptoms } & \multicolumn{1}{c}{ Signs } \\
Was breathlessness a symptom? If so: & \\
Breathless at rest? & Temperature $>37 \cdot 5^{\circ} \mathrm{C} ?$ \\
Orthopnoea? & Cyanosis? \\
Breathless at night? & Hepatomegaly? \\
Sudden deterioration? & Sweating? \\
Gradual decline? & Peripheral vasoconstriction? \\
Intermittent with trouble-free intervals? & Peripheral vasodilatation? \\
If nocturnal dyspnoea? & Tachycardia > 100 min? \\
Woken from sleep? & Systolic BP < 100 mmHg? \\
Accompanied by coughing? & Enlarged heart? \\
Relieved by sitting up? & Raised JVP? \\
Relieved by getting out of bed? & Ankle oedema? \\
Was cough a symptom? If so: & Added heart sounds \\
Productive on admission? & (3rd, 4th or gallop)? \\
History recent sputum purulence? & Respiratory frequency $>$ 20/min? \\
Long-standing? & Bronchial breathing? \\
Haemoptysis? & Local increase vocal resonance? \\
Ankle swelling? & Localized reduction breath sounds? \\
Calf pain? & Localized rhonchi? \\
Was chest pain a symptom? If so: & Generalized rhonchi? \\
Present on admission? & Localized crepitations? \\
Pleuritic? & Generalized crepitations? \\
Retrosternal? & Bilateral basal crepitations? \\
Radiation to arms, throat or jaw? & Pleural rub? \\
Nausea? & \\
Preceding history angina? & \\
Relief only by diamorphine? & \\
\hline
\end{tabular}


by Swinscow (1976a). Only patients treated for single diagnoses were analysed in this way.

When analysing the 2 periods of time (summer and winter) for the incidence of different categories of treatment, the analysis has been either by a $2 \times 2$ contingency table as described or where it seemed more logical to present the results for the different categories of treatment as percentages or proportions of some other figure (see results section), the authors tested the results for significance using the standard error of a difference between percentages as described by Swinscow (1976b).

\section{Results}

\section{Treatment given (Table 2)}

Of 352 acute medical admissions, 104 were treated for breathlessness during the summer survey period while during the winter period the proportion was 73 patients out of 355 . This difference is significant $(P<0.01)$ and surprising.

More patients received bronchodilator drugs during the winter period than the summer. Otherwise there was no significant difference in treatment patterns in the summer as compared with the winter.

Combinations of treatment, which it is suggested reflect diagnostic uncertainty, were more common $(P<0.01)$ in the winter (29 out of 73 patients) than in the summer (19 out of 104). When the combination treatments are analysed further it is found that combinations involving treatment for chest infection are more common than combinations involving other treatments in both the summer $(P<0.001$ for comparison with heart failure, embolism and asthma) and the winter $(P<0.05$ when comparing with heart failure; $P<0.01$ comparing with asthma and $P<0.001$ comparing with pulmonary embolism).

If combinations of treatment represent diagnostic uncertainty, it is possible to derive an 'index of uncertainty' for each treatment category by calculating the proportion of the total number of patients receiving any given treatment who also receive other treatments. For example, if one considers treatment for heart failure during the summer period, the total number of patients receiving treatment for heart failure was 64 of whom 10 also received some additional treatment giving an index of uncertainty of

$$
\frac{10}{64} \times 100=16 \% \text {. }
$$

The corresponding index for the treatment of infection during the summer period was

$$
\frac{19}{37} \times 100=51 \%
$$

implying greater uncertainty in the diagnosis of chest infection than of heart failure. This 'index of

\begin{tabular}{|c|c|c|c|c|c|}
\hline & Summer & & Winter & & Total \\
\hline \multicolumn{6}{|l|}{ One treatment regime only: } \\
\hline Heart failure & 54 & & 29 & & 83 \\
\hline Chest infection & 18 & & 8 & & 26 \\
\hline Pulmonary embolism & 8 & & 4 & & 12 \\
\hline \multirow[t]{2}{*}{ Asthma } & 5 & & 3 & & 8 \\
\hline & 85 & & 44 & & 129 \\
\hline More than one treatment regime & 19 & & 29 & & 48 \\
\hline Total & 104 & & 73 & & 177 \\
\hline Combinations of more than one treatment regime: & \multicolumn{3}{|c|}{ Summer } & \multicolumn{2}{|l|}{ Winter } \\
\hline Combinations involving heart failure & \multirow{2}{*}{\multicolumn{3}{|c|}{$\begin{array}{l}10 \\
19\end{array}$}} & 18 & \\
\hline Combinations involving chest infection & & & & 26 & \\
\hline Combinations involving pulmonary embolism & \multicolumn{3}{|c|}{3} & 3 & \\
\hline Combinations involving asthma & \multicolumn{3}{|c|}{8} & 16 & \\
\hline \multicolumn{6}{|l|}{ Total nos of patients receiving treatment for: } \\
\hline Heart failure & \multicolumn{3}{|c|}{64} & \multicolumn{2}{|l|}{47} \\
\hline Chest infection & \multirow{2}{*}{\multicolumn{3}{|c|}{37}} & \multicolumn{2}{|l|}{34} \\
\hline Pulmonary embolism & & & & \multicolumn{2}{|l|}{7} \\
\hline Asthma & \multicolumn{3}{|c|}{13} & \multicolumn{2}{|l|}{19} \\
\hline \multicolumn{6}{|l|}{ 'Index of Uncertainty' (see text) } \\
\hline Heart failure & \multirow{4}{*}{\multicolumn{3}{|c|}{$\begin{array}{r}10 / 64(16 \%) \\
19 / 37(51 \%) \\
3 / 11(27 \%) \\
8 / 13(62 \%)\end{array}$}} & \multirow{4}{*}{\multicolumn{2}{|c|}{$\begin{array}{c}18 / 47(38 \%) \\
26 / 34(76 \%) \\
3 / 7(43 \%) \\
16 / 20(80 \%)\end{array}$}} \\
\hline Chest infection & & & & & \\
\hline Pulmonary embolism & & & & & \\
\hline Asthma & & & & & \\
\hline
\end{tabular}

TABLE 2. Number of patients in various treatment categories in the survey periods 
uncertainty' is significantly lower for heart failure in both summer $(16 \%)$ and winter $(38 \%)$ than for infection (51\% summer, $P<0.001 ; 76 \%$ winter, $P<0.01)$ or for asthma $(62 \%$ summer, $P<0.001$, $80 \%$ winter $P<0.01$ ). None of the other comparisons was significantly different but when the winter and summer periods were compared, the 'index of uncertainty' was higher in winter for both infection $(P<0.05)$ and failure $(P<0.01)$ than during summer.

\section{Discriminating signs and symptoms}

Patients who received treatment for a single diagnosis in both study periods were combined for analysis. Using a $2 \times 2$ contingency table each treatment category was compared with the rest of the treatment categories combined for the frequency with which each sign and symptom was recorded in the case notes.

Heart failure. The following features of the history were regarded as being of discriminatory value $(P<0.05)$ by the admitting doctors as judged by the differential frequency with which they were recorded in the case notes. Patients treated for heart failure alone were more likely to have complained of sudden deterioration $(<0.05)$, orthopnoea $(<0.02)$, nocturnal dyspnoea $(<0.001)$, chest pain on admission $(<0.01)$ and pain of a cardiac distribution (retrosternal radiating to arms or throat, $<0.001)$, nausea $(<0.01)$ and sweating $(<0.01)$. They were more likely to have a past history of angina $(<0.05)$, hypertension $(<0.05)$ and myocardial infarction $(<0.05)$.

Signs reported significantly more often were sweating $(<0.05)$, peripheral vasoconstriction $(<0.01)$, cardiac enlargement $(<0.01)$, neck vein engorgement $(<0.001)$ ankle oedema $(<0.01)$, added heart sounds (3rd, 4th or gallop rhythm) $(<0.01)$ and bilateral basal crepitations $(<0.001)$.

Chest infection. Patients treated for infection alone were more likely to give a history of gradual decline $(<0.05)$, recent cough $(<0.01)$, sputum production on admission $(<0.01)$, recent sputum purulence $(<0.001)$ and frank haemoptysis $(<0.01)$ but, surprisingly, a history of simple chronic bronchitis (Report to the Medical Research Council, 1965) was no more common in patients treated for infection.

Signs recorded significantly more often in patients treated for infection were body temperature above $37.5^{\circ} \mathrm{C}(<0.001)$, dehydration $(<0.05)$, tachycardia $>100 /$ min $(<0.05)$, local reduction in breath sounds $(<0.02)$ local crepitations $(<0.001)$, localized rhonchi $(<0.05)$ and signs of consolidation (increased vocal resonance and bronchial breathing $(<0.01)$.

Pulmonary embolism. The decision to treat for pulmonary embolism was made on the presence of calf pain $(<0.001)$ and/or pleuritic chest pais $(<0.001)$. The only sign recorded significantly mor\& frequently in this group was the presence of calf. tenderness $(<0.02)$.

Asthma. Intermittent breathlessness with trouble free intervals was regarded as an important dise criminatory point in the history as judged by the differential frequency with which it was recorded i the case notes $(<0.001)$ as was a history of non? productive cough $(<0.01)$.

A respiratory frequency $>20 / \mathrm{min}(<0.02)$ and the presence of generalized wheezes $(<0.001)$ were the only signs recorded more frequently in patients. treated for asthma alone than for the other groups

In addition to the above comparisons, comparisoris between pairs of treatment groups, for example patients treated for heart failure alone as compared with patients treated for infection alone, gives somg additional information. Thus, a preceding histor of angina or hypertension was equally common in patients treated for heart failure and patients treateg for infection. Patients treated for heart failure were more likely to be breathless at rest $(<0.05)$ and those treated for chest infection to have pleuritic ches pain $(<0.02)$. Orthopnoea was recorded with equal. frequency in the 2 groups as were wheezes, genegabo ized crepitations and added heart sounds (t sound, fourth sound and gallop rhythm).

A comparison of patients treated for infection alone with those treated for embolism alone showe $\widehat{ }$ that the decision to treat for pulmonary embolisro rather than infection was made entirely on the history? Embolism was favoured if deterioration had beef sudden $(<0.05)$ and if chest pain was presen $(<0.05)$, particularly if it was pleuritic $(<0.01)$ Haemoptysis was equally common in the 2 groups? Importance was attached to a past history of myes cardial infarction (favouring embolism, $P<0.05 \Phi$. and to the presence of calf pain $(<0.05)$ but not to ankle swelling. No physical sign was recorded more. frequently in one treatment group than the other and this included the presence of a body temperature $>37 \cdot 5^{\circ} \mathrm{C}$.

A comparison of patients treated for heart failure alone with those treated for pulmonary embolisn. alone showed no significant difference in thes frequency of a history of haemoptysis in the 2 groups.

\section{Role of the chest X-ray}

Sixty-four patients $(62.5 \%)$ had chest X-rays done before initiation of treatment during summer and $50(68.5 \%)$ during winter. Of these X-rays the pro? portion which the treating doctor claimed had in fluenced their choice of treatment was $63 \%$ in the summer and $64 \%$ in the winter. Thirty patients in the summer $(28.8 \%)$ and 10 in the winter $(13.7 \%)$ has 
X-rays done only after treatment had been initiated while in 9 patients in the summer and 13 in the winter there was no record of any chest X-ray having been done.

\section{Discussion}

Using the treatment prescribed as an index of the operational diagnosis, the authors set out to see how the text book stereotypes of the diagnostic characteristics of the main causes of breathlessness influenced management. By surveying their doctor's diagnostic and prescribing habits they have determined how frequently the breathless patient receives treatment for a single disease and, when this is the case, they have determined the symptoms and signs on which the admitting doctors rely most in making their decisions. It was thought likely that diagnostic uncertainty would be reflected in multiple lines of treatment or frequent changes in therapy. This is not to deny that in some patients multiple pathology exists, for example the patient with chest infection frequently has an element of reversible airflow obstruction, and there are no means of proving this assumption on the basis of this survey. However, in support of this hypothesis the authors find that as the incidence of combination of treatments increases so does the number of treatments used in the combinations. Thus, in summer the overall use of combination treatment is less common than in the winter. In summer $18 \%$ of breathless patients receive 2 lines of treatment and $2 \%$ more than 2 , whereas in winter $33 \%$ of patients receive 2 lines of treatment and $7 \%$ more than 2. If this is to be explained on the basis of multiple pathology it means that not only is multiple pathology more frequent in winter than summer but also that there is a disproportionate increase in the number of patients with at least 3 separate causes for their breathlessness. The alternative is to view the incidence of combination treatment as a measure of diagnostic uncertainty and increasing numbers of treatments as suggesting increasing uncertainty. This does not imply any judgement as to the appropriateness or inappropriateness of treatment in any particular case but it provides a basis for further studies to determine the reasons for the differences in management herein described.

The signs and symptoms used by junior doctors to decide on initial treatment must reflect their previous teaching. As judged by the frequency with which they were recorded in the case notes, bilateral basal crepitations were equated with heart failure. The belief that such sounds simply represent intraalveolar oedema is no longer tenable (Forgacs, 1969), but clinical training does not yet reflect this. Fever is a non-specific sign, being a feature of tissue infarction as well as infection while sputum purul- ence need not denote infection, but these pieces of evidence and the presence of localized crepitations were used as indicators of chest infection by the authors' doctors. The frequency of chest pain in the group treated for heart failure shows that doctors are more ready to diagnose heart failure in patients whom they believe may have had a myocardial infarction. On the other hand, the presence of orthopnoea did not influence the diagnosis of heart failure as opposed to chest infection, nor was haemoptysis taken as an indicator of the need to treat for pulmonary embolism.

In emergency medicine, history taking and examination by admitting doctors, who are usually junior, invariably determines the initial treatment. However, in this study, changes in management as doubt increased or as other doctors became involved, resulted in the addition of further drugs and none of the treatments which had been initiated by admitting doctors was withdrawn by their seniors over the next few days. Assessment by senior physicians of the symptoms and signs used by their juniors to make treatment decisions might reveal areas in which more research is needed to evaluate their true discriminating power or possibly areas in which further training of their junior staff is required. Perhaps training should also stress the need to collect more evidence in that the authors' admitting doctors never recorded the results of sputum inspection (although they usually enquired about it from the patient) and seldom measured peak expiratory flow rate and forced vital capacity.

This paper has been concerned with clinical assessment since this influences emergency care and since the tests requested by admitting doctors clearly reflect the diagnosis already reached. The authors are now trying to assess the contribution of investigative aids to diagnosis because many doctors place great faith in them and because even relatively simple investigations done frequently but unnecessarily use considerable resources (Wilcox and Mitchell, 1977). The questions which need to be answered are whether the chest radiograph is a reliable aid to the diagnosis of left ventricular failure? Does a lung scan really influence the management of patients in whom pulmonary embolism is suspected on clinical grounds or are the majority of patients anticoagulated on these clinical suspicions irrespective of the result of the scan? Does the morbidity and mortality in patients managed without the benefit of investigation differ significantly from those who receive it? By studying the early management of the common medical emergency conditions which bring patients into hospital one would find that the task of the admitting doctor to whom one delegated emergency care is much more difficult than the standard text books would suggest. Once one 
admits this, one can begin to reappraise these stereotyped descriptions and try to identify symptoms, signs and investigations which have true discriminating power.

\section{Acknowledgments}

We thank the physicians at the General Hospital, Nottingham, for permission to study their patients and to the junior medical staff for their co-operation.

\section{References}

Crofton, J. \& Douglas, A. (1975) Respiratory Diseases, 2nd edn, p. 444. Blackwell Scientific Publications, Oxford. ForgACs, P. (1969) Lung sounds. British Journal of Diseases of the Chest, 63, 1.
Houston, J.C., JoIner, C.L. \& TrounCe, J.R. (1975) $A \geq$ Short Text-Book of Medicine. English Universities Press, London.

MACLEOD, J. (Ed.) (1977) Davidson's Principles and Practice $\hookrightarrow$ of Medicine. Churchill Livingstone, Edinburgh.

RePORT TO THE MEDICAL RESEARCH Council by their $\stackrel{\vec{\rho}}{\rightarrow}$ committee on the aetiology of chronic bronchitis (1965) Lancet, i, 775.

SwINScow, T.D.V. (1976a) Statistics at Square One, p. 49. British Medical Journal, London.

SwinsCow, T.D.V. (1976b) Statistics at Square One, pp. 28-30. British Medical Journal, London.

WILCoX, R.G. \& Mirchell, J.R.A. (1977) Intravenous urography in the management of acute retention. Lancet, $\vec{O}$ i, 1247.

YATES, F. (1934) Contingency tables involving small numbers $\vec{\omega}$ and the Chi-squared test. Journal of the Royal Statistical Society, 1 (Suppl.), 217. 